\title{
Efeitos do exercício na redução do risco de quedas em mulheres idosas com osteoporose
}

\author{
Effects of exercise in reducing the risk of falls in elderly women with osteoporosis
}

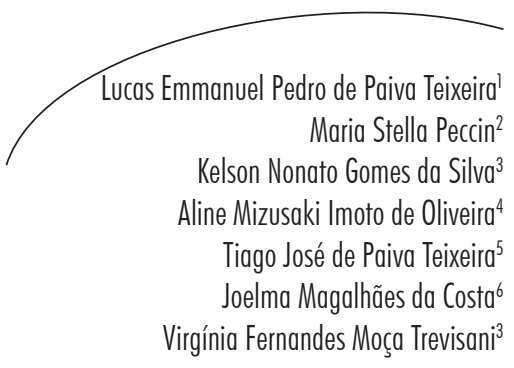

\section{Resumo}

Objetivo: Avaliar o efeito de um programa de treinamento de força muscular progressiva e sensório-motor, no equilíbrio e na redução do risco de quedas em mulheres com osteoporose. Método: Foram selecionadas 82 mulheres sedentárias com idade entre 60 e 75 anos com osteoporose, randomizadas em dois grupos: grupo intervenção, composto por 41 pacientes submetidas a 18 semanas de treinamento de força muscular progressiva do quadríceps (a 50\%, 60\%, 70\% até 80\% de 1-RM - uma repetição máxima), e de propriocepção, associado ao tratamento clínico medicamentoso para osteoporose; e grupo controle, também composto por 41 pacientes que receberam apenas o tratamento clínico medicamentoso. O equilíbrio e o risco de quedas foram avaliados por meio da Escala de Equilíbrio de Berg (BBS) e do cálculo do Índice de Quedas (IQ). O número de quedas foi avaliado seis meses antes do início da pesquisa e nos seis meses seguintes. Resultados: 69 pacientes concluíram a pesquisa. O programa promoveu melhora no equilíbrio funcional $(\mathrm{p}<0,001)$ demonstrado pela BBS, redução do risco de quedas calculado pelo escore geral do IQ $(\mathrm{p}<0,004)$ e ainda uma redução do número total de quedas, que no grupo experimental foram significativamente menores que no grupo controle $(\mathrm{p}<0,001)$. Conclusão: A associação do treinamento de força progressiva para quadríceps e propriocepção é eficaz na prevenção de quedas e redução de seus fatores de risco, e o modelo para previsão quantitativa do risco de quedas pode ser útil na identificação do risco de quedas em pessoas idosas. Registro ensaio clínico: 082213-ANZCTR.

\footnotetext{
Departamento de Fisioterapia. Universidade Federal do Amazonas. Manaus, AM, Brasil

Departamento de Ciências do Movimento Humano. Universidade Federal de São Paulo. Santos, SP, Brasil.

Programa de Pós-graduação em Medicina Interna e Terapêutica. Universidade Federal de São Paulo. São Paulo, SP, Brasil.

4 Serviço de Fisioterapia. Hospital das Forças Armadas. Brasília, DF, Brasil.

Residência em Reumatologia. Universidade Santo Amaro. São Paulo, SP, Brasil.

Departamento de Fisioterapia. Centro Universitário do Norte. Manaus, AM, Brasil.
}

\section{Palavras-chave:}

Osteoporose. Quedas.

Prevenção. Exercícios. 


\section{Abstract}

Objective: This study aims to evaluate the effect of progressive muscular strength and proprioception training program on the balance and decrease in the risk of falls in women with osteoporosis. Method: 82 sedentary women aged 60-75 years with osteoporosis were selected and randomized in two groups: the intervention group comprised 41 patients submitted to an 18-week progressive muscular strength and proprioception training of quadriceps associated to drug therapy for osteoporosis; and control group, formed by 41 patients submitted to drug therapy only. The balance and fall risk were evaluated using the Balance Berg Scale (BBS) and calculating the Fall Index (FI). The number of fall was assessed six months before the research and the following six months. Results: 69 patients completed the research. The program improved the functional balance $(p<0.001)$ shown by BBS, decreased the risk of falls evidenced by the general score of FI $(\mathrm{p}<0.004)$ and also a decrease in the total number of falls. The experimental group showed significant lower number of falls when compared to the control group $(\mathrm{p}<0.001)$. Conclusion: The association of progressive strength of quadriceps and proprioceptive training is effective to prevent falls and reduce risk factors. The quantitative model to predict risk of falls might be useful to identify the risk of falls in the elderly. Trial registration: 082213-ANZCTR.
Key words: Osteoporosis. Falls. Prevention. Exercises.

\section{INTRODUÇÃO}

Queda é definida como sendo a ocorrência de um evento não intencional que leva uma pessoa inadvertidamente a cair ao chão em um mesmo nível ou em outro inferior.

Para Tinett \& Willians, ${ }^{2}$ a incidência de quedas em mulheres com idade acima de 72 anos é de $49 \%$, enquanto Campbell et al. ${ }^{3}$ sinalizam uma incidência de 53\% em mulheres com idade superior a 80 anos. No mesmo estudo, a incidência de fraturas em pessoas com 70 anos ou mais foi de $10 \%$ e a de ferimentos graves causados por quedas foi de $16 \%$.

A alta incidência de quedas reflete-se em um grande número de mortes. Pesquisa realizada no Estado de São Paulo mostrou que no ano de 2007 as quedas foram responsáveis por 1.328 mortes no Estado, representando 31,8\% do total de mortes por causa externa em idosos, com coeficiente de 31/100.000 habitantes. ${ }^{4}$ Os idosos que caem sucessivamente são expostos a um risco ainda maior de sofrer fraturas e mortes. ${ }^{5,6}$
A osteoporose com baixa densidade óssea expõe as pessoas mais idosas que caem a um alto risco de sustentar fraturas. ${ }^{7,8}$ Uma primeira fratura de quadril é associada com um aumento de duas vezes e meia no risco de uma fratura subsequente, ${ }^{9}$ com alto nível de morbidade e mortalidade..$^{10} \mathrm{~A}$ redução da densidade óssea, associada a uma redução da força muscular e alterações do sistema somatossensorial, expõe as mulheres idosas a um risco aumentado de sofrer fraturas. Uma redução de aproximadamente $30 \%$ na força é encontrada entre pessoas de 50 e 70 anos de idade. Tais mudanças são mais comuns em mulheres do que em homens; mais em membros inferiores do que em membros superiores, sendo que muito desta redução de força se deve a uma atrofia seletiva das fibras musculares do tipo IIB. ${ }^{11}$

A prevenção de quedas é área de grande importância em pesquisas em saúde do idoso. ${ }^{3}$ Há evidência crescente de que intervenções de exercício específicas podem reduzir fatores de risco durante quedas e quedas atuais em idosos, ${ }^{12-15}$ mas um modelo capaz de avaliar o 
risco de quedas em mulheres idosas é necessário. O modelo proposto por Shumway-Cook et al. ${ }^{16}$ é o de maior especificidade e sensibilidade, mas ainda carece de mais estudos que o indiquem como um preditor efetivo do risco de quedas.

Desta forma, este estudo teve por objetivo investigar os efeitos do treinamento sensóriomotor e de força muscular na redução do risco de quedas, além de avaliar a relevância da aplicação do índice de quedas como preditor do risco de cair.

\section{MATERIAL E MÉTODOS}

Para esta pesquisa, foram selecionadas 82 mulheres com idade entre 60 e 75 anos, cadastradas no setor de Diagnóstico por Imagem do Serviço de Reumatologia do Ambulatório de Especialidades de Interlagos, São Paulo, SP, Brasil, no período de agosto de 2007 a outubro de 2009. Foram incluídas somente pacientes com diagnóstico de osteoporose, de acordo com a Organização Mundial da Saúde (OMS) com Densidade Mineral Óssea (BMD) T-score - 2,5 de divergência standard (SD) na coluna lombar, colo do fêmur ou região femoral total.

Foram excluídas mulheres com deficiência visual, sem possibilidade de correção prévia; com deficiência auditiva grave com alteração vestibular de estado clínico importante; que usam dispositivos de auxílio da marcha (órteses ou próteses); que planejaram viagens que exigissem ausência por duas semanas consecutivas durante as 18 semanas da pesquisa; que apresentassem contraindicações absolutas ou relativas ao exercício físico, de acordo com o Colégio Americano de Medicina do Esporte. A realização da pesquisa foi aprovada pelo Comitê de Ética em Pesquisa (CEP) da Universidade Federal de São Paulo, protocolo no 1910/2006, e o ensaio clínico foi registrado no Australian New Zealand Clinical Trials Registry (ANZCTR), sob número 082213.

As pacientes selecionadas pelos critérios de inclusão e exclusão e que aceitaram participar da pesquisa assinaram o Termo de Consentimento Livre e Esclarecido (TCLE). A randomização foi feita por computador e os números sequenciais foram mantidos em envelopes opacos, não translúcidos e lacrados, sendo alocados para um dos dois grupos baseado no Consort.

Das 758 densitometrias ósseas analisadas no serviço de Diagnóstico por Imagem do Ambulatório de Especialidades de Interlagos, 284 eram positivas para o diagnóstico de osteoporose, sendo que 162 estavam dentro da faixa etária proposta pela pesquisa e 82 foram incluídas por preencherem os critérios de inclusão exigidos. Das 82 pacientes randomizadas, 69 concluíram a pesquisa, sendo 37 no G1 e 32 no G2.

As voluntárias formaram dois grupos: um primeiro, chamado de G1, composto por 41 pacientes submetidas a 18 semanas de treinamento de fortalecimento muscular progressivo e de propriocepção associado ao tratamento clínico medicamentoso para osteoporose; o segundo grupo, chamado de G2, também composto por 41 pacientes, recebeu apenas o tratamento clínico medicamentoso convencional (figura 1). 


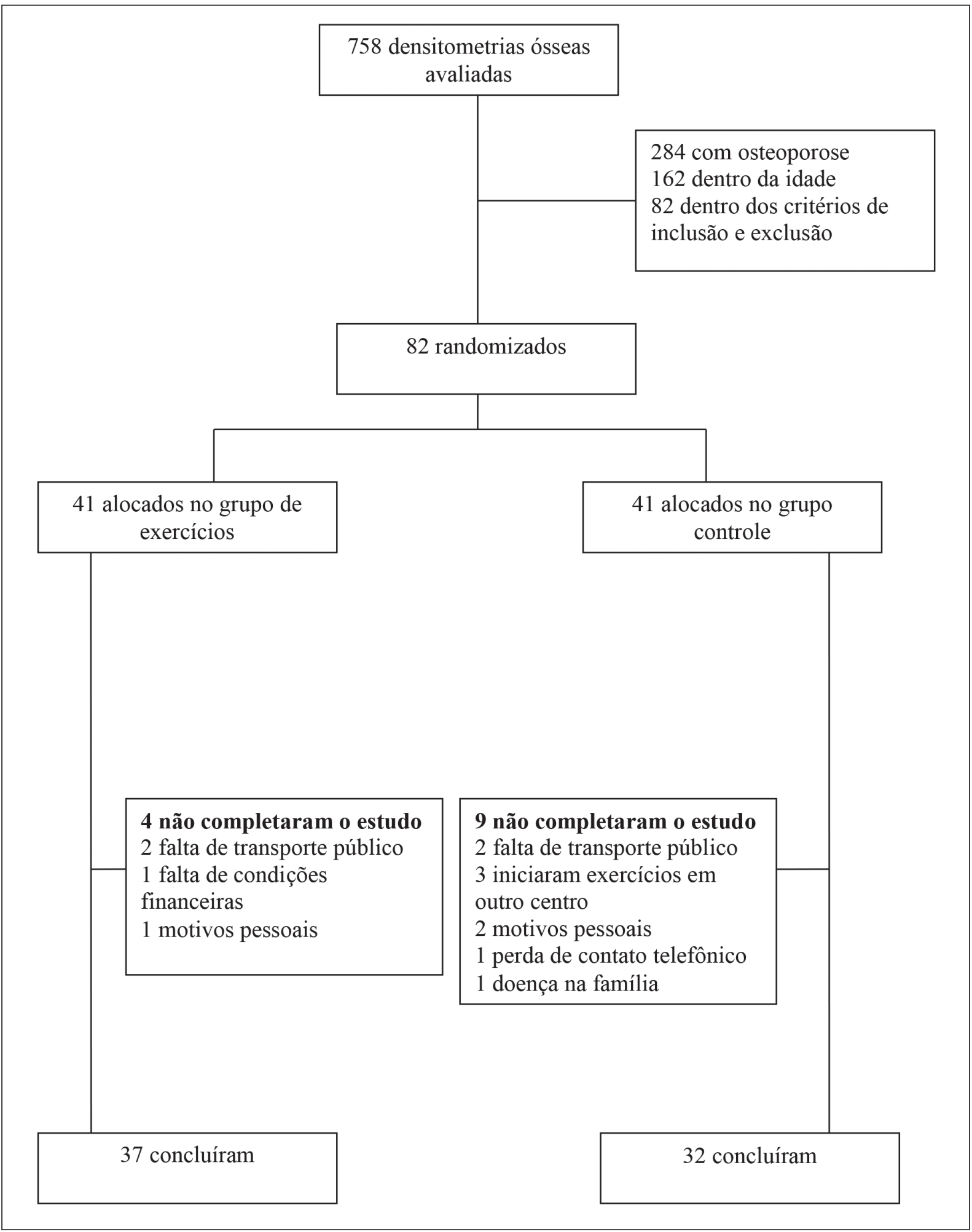

Figura 1 - Organograma da análise de inclusão e exclusão, randomização, formação dos grupos, perdas e pacientes que concluíram o programa. São Paulo-SP, 2007-2009. 
Avaliação

Durante a avaliação, todas as pacientes tinham seus dados pessoais e clínicos anotados pelo médico responsável pela avaliação dos critérios de inclusão e exclusão. Todas as pacientes foram avaliadas por fisioterapeuta cego para o grupo a que cada indivíduo pertencia. Foram avaliadas a qualidade de vida, a capacidade funcional, o risco e o número de quedas.

O equilíbrio foi avaliado por meio da Escala de Equilíbrio de Berg, que avalia o desempenho do equilíbrio funcional em 14 itens comuns à vida diária. Esta escala tem uma pontuação máxima que pode ser alcançada de 56 pontos e cada item possui uma escala ordinal de cinco alternativas que variam de 0 a 4 pontos. ${ }^{14,15}$

Foi utilizado ainda o modelo para previsão quantitativa do risco de quedas em idosos que estabelece a relação entre a Escala de Equilíbrio de Berg e o risco de quedas, que varia de 0 a $100 \%$, desenvolvido por Shumway-Cook et al. ${ }^{16}$ Nesse modelo, a sensibilidade da escala foi de $91 \%$ e a especificidade, $82 \%$. A probabilidade de queda aumenta com a diminuição da pontuação da Escala de Equilíbrio de Berg, numa relação não linear. Na amplitude de 56 a 54, cada ponto a menos é associado a um aumento de 3 a $4 \%$ no risco de quedas. De 54 a 46, a alteração de um ponto é associada ao aumento de 6 a $8 \%$, e abaixo de 36 pontos, o risco de quedas é quase de $100 \%$. O modelo incluiu o escore da BBS e a história de desequilíbrio, sendo: 0 para nenhuma história de desequilíbrio e 1 para história positiva de desequilíbrio dentro dos seis meses prévios. O modelo é relacionado à probabilidade de cair, pela equação seguinte: probabilidade $=100 \% \mathrm{X}$ exp (10,46 - 0,25 X Berg Balance Scale score + 2,32 X história de desequilíbrio)/ $[1+\exp (10,46$ - 0,25 X Berg Balance Scale score + 2,32 X história de desequilíbrio)]. ${ }^{16}$
O número de quedas foi avaliado por meio da monitorização do relato imediato das quedas dos pacientes de ambos os grupos durante 24 semanas. Os pacientes também foram questionados se eles tiveram quedas nos seis meses que antecederam ao estudo.

Intervenção

O protocolo foi disposto em circuito contendo: 1) aquecimento de 5-10 minutos, com bicicleta estacionária, exercícios de alongamento estático para membros inferiores e superiores, região lombar, cervical e região do tronco, com três repetições para cada músculo ou grupo muscular, mantendo por 30 segundos, com intervalos de 30 segundos entre as séries; 2) os exercícios funcionais (propriocepção e equilíbrio) realizados em circuito seguem uma ordem de evolução iniciando com solos estáveis passando para solos instáveis, treino de marcha sem obstáculo evoluindo para marcha com obstáculos, olhos abertos para olhos fechados, baixa velocidade e de acordo com a evolução aumenta-se a velocidade na execução dos exercícios, treino bipodal evoluindo para unipodal, sendo utilizados também recursos como balanço, cama elástica, pranchas proprioceptivas seguindo sempre a mesma evolução; 3) exercícios de fortalecimento em cadeira extensora variando a carga até $80 \%$ de $1-\mathrm{RM}$, seguindo protocolo de duas semanas de adaptação com caneleiras de 1 a $2 \mathrm{~kg}$, e então seguindo para progressão de $50 \%$, $60 \%, 70 \%$ até $80 \%$ de $1-$ RM. $^{17,18}$

\section{Análise estatística}

Para o cálculo de tamanho de amostra, foram utilizados dados de pesquisas anteriores. Com base no número de quedas para aplicar $t$ Student, feito por meio do software Statistical Analisys System (SAS), versão 8.02, considerou-se o nível de significância de $\alpha=0,05$, com poder estatístico 
$1-\beta=0,80$. Utilizando-se esses parâmetros, foi calculado um número mínimo de 35 pacientes para cada grupo.

Para verificar o pressuposto de normalidade na distribuição dos dados, foi empregada a prova estatística de Shapiro-Wilk, bem como se fez a inspeção dos gráficos de quantis (qqplot). Uma vez que a distribuição das variáveis estudadas não podia ser aproximada pela distribuição normal, foram calculados a mediana e os quartis 1 e 3 para descrever as variáveis estudadas.

A significância da influência do momento do tratamento (pré e pós-intervenção) e dos grupos (controle e experimental) foi avaliada por meio da prova de hipótese não paramétrica descrita por Brunner et al. ${ }^{19} \mathrm{~A}$ significância estatística foi aceita em $\mathrm{p} \leq 0,05$. Todo o processamento estatístico foi realizado no ambiente estatístico R (versão, 2.6.2; $\mathrm{R}$ Foundation for Statistical Computing, Viena, Áustria).

\section{RESULTADOS}

As características basais de ambos os grupos eram semelhantes em relação a idade, densidade mineral óssea, história de fraturas, tratamento para osteoporose, uso de diuréticos, hipnóticos e antidepressivos nos seis meses anteriores ao início do estudo. Apresentaram ainda frequência de $82 \pm 5,83 \%$ nas sessões de treinamento (mínimo de $75 \%$ e máximo de $100 \%$ ).

De acordo com o descrito na figura 2, observase que na avaliação inicial o escore para BBS era estatisticamente semelhante na comparação entre os grupos.

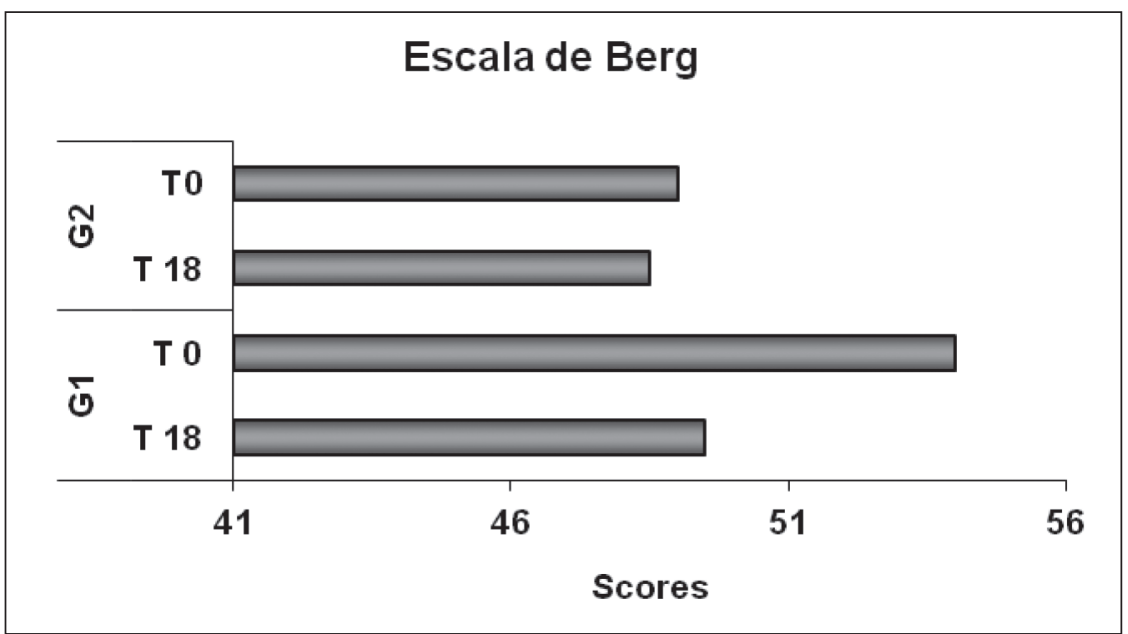

Figura 2 - Escala de Berg comparando os períodos anteriores ao tratamento e após as 18 semanas de tratamento, nos grupos intervenção (G1) e controle (G2). Variáveis "componente rotacional" e "base diminuída" (tabela 1). São Paulo, SP, 2007-2009. 
O escore geral da Escala de Berg (tabela 1) apresentou aumento significativo no grupo experimental $(p<0,001)$, sendo os valores de pós-treinamento significativamente maiores no grupo experimental em relação ao grupo controle $(p<0,001)$. As variáveis "atividade física", "componente rotacional" e "base diminuída" apresentaram aumento significativo tanto na comparação com a admissão como na comparação com o controle $(\mathrm{p} \leq 0,003)$. Não houve diferenças estatística nem clinicamente significativas nos quesitos transferência e provas estáticas.

Tabela 1 - Descrição e comparação das variáveis obtidas pela Escala de Berg. São Paulo-SP, 2007-2009.

\begin{tabular}{lcccc}
\hline \multirow{2}{*}{ Variáveis } & \multicolumn{2}{c}{ Controle } & \multicolumn{2}{c}{ Experimental } \\
\cline { 2 - 5 } & Pré & Pós & Pré & Pós \\
\hline Transferência & $10[9 ; 10]$ & $10[9 ; 11]$ & $10[10 ; 10]$ & $10[10 ; 10]$ \\
Provas estáticas & $10[10 ; 10]$ & $10[10 ; 10]$ & $10[10 ; 10]$ & $10[10 ; 11]$ \\
Atividade física & $2[2 ; 3]$ & $2[2 ; 3]$ & $2,5[2 ; 3]$ & $4[4 ; 4]^{*+}$ \\
Capacidade residual & $9[9 ; 10]$ & $9[9 ; 10]$ & $9[9 ; 10]$ & $12[12 ; 12]^{*+}$ \\
Base diminuída & $10[9 ; 10,75]$ & $10[9 ; 11]$ & $10[9 ; 11]$ & $12[11 ; 12]^{*+}$ \\
Berg & $48,5[47 ; 50]$ & $49[48 ; 50]$ & $49,5[47,5 ; 54]$ & $54[51 ; 54]^{* \dagger}$ \\
\hline
\end{tabular}

Dados apresentados na forma de mediana [quartil 1; quartil 3].

*Indica diferença estatisticamente significativa em relação ao grupo controle para o mesmo momento.

†Indica diferença estatisticamente significativa em relação ao momento pré-experimento.

Apesar de a mudança numérica ser pequena, ela é consistente; muitas pessoas no grupo experimental apresentaram aumento na pontuação, por isso mesmo um valor pequeno. Isso pode ser visto na aplicação do modelo de Shumway-Cook et al., ${ }^{16}$ apresentado na tabela 2 .
O escore geral do Índice de Quedas (IQ) (tabela 2) apresentou aumento significativo no grupo experimental $(\mathrm{p}<0,001)$, sendo os valores de pós-treinamento significativamente maiores no grupo experimental em relação ao grupo controle $(\mathrm{p}<0,004)$.

Tabela 2 - Descrição e comparação das variáveis obtidas por meio do modelo para previsão quantitativa do risco de quedas em idosos, que estabelece a relação entre a Escala de Equilíbrio de Berg e o risco de quedas, que varia de 0 a 100\%, desenvolvido por Shumway-Cook et al. ${ }^{16}$ São Paulo-SP, 2007-2009.

\begin{tabular}{ccccc}
\hline \multirow{2}{*}{ Variáveis } & \multicolumn{2}{c}{ Controle } & \multicolumn{2}{c}{ Experimental } \\
\cline { 2 - 5 } & Pré & Pós & Pré & Pós \\
\hline \multirow{2}{*}{ Risco de quedas $(\%)$} & 44,52 & 38,46 & 38,46 & 2,86 \\
& {$[22,79 ; 56,95]$} & {$[27,48 ; 62,95]$} & {$[22,79 ; 56,95]$} & {$[2,82 ; 4,56]^{*+}$} \\
\hline
\end{tabular}

Dados apresentados na forma de mediana [quartil 1; quartil 3].

*Indica diferença estatisticamente significativa em relação ao grupo controle para o mesmo momento.

†Indica diferença estatisticamente significativa em relação ao momento pré-experimento. 
Reforçando esses dados, foi encontrada redução do risco de quedas calculado por meio do odds ratio, onde a chance de uma pessoa que fez o tratamento sofrer queda é apenas $21,4 \%$ da chance de uma pessoa que não fez o tratamento. Ou a chance de uma pessoa que não faz o tratamento sofrer queda é 4,67 vezes maior do que uma pessoa que fez o tratamento. $\mathrm{O}$ valor é semelhante ao encontrado neste estudo, quando analisado o número total de quedas, sendo a chance de quedas no grupo controle 3,8 vezes maior do que no que recebeu tratamento.
Com base nos resultados positivos do protocolo aplicado sobre a condição física, observou-se expressiva redução no número total de quedas (figura 3), verificando-se redução significativa entre pré e pós-treinamento para o grupo experimental $(\mathrm{p}<0,001)$. Além disso, os valores de pós-treinamento do grupo experimental foram significativamente menores que os apresentados pelo grupo controle $(\mathrm{p}<0,001)$.

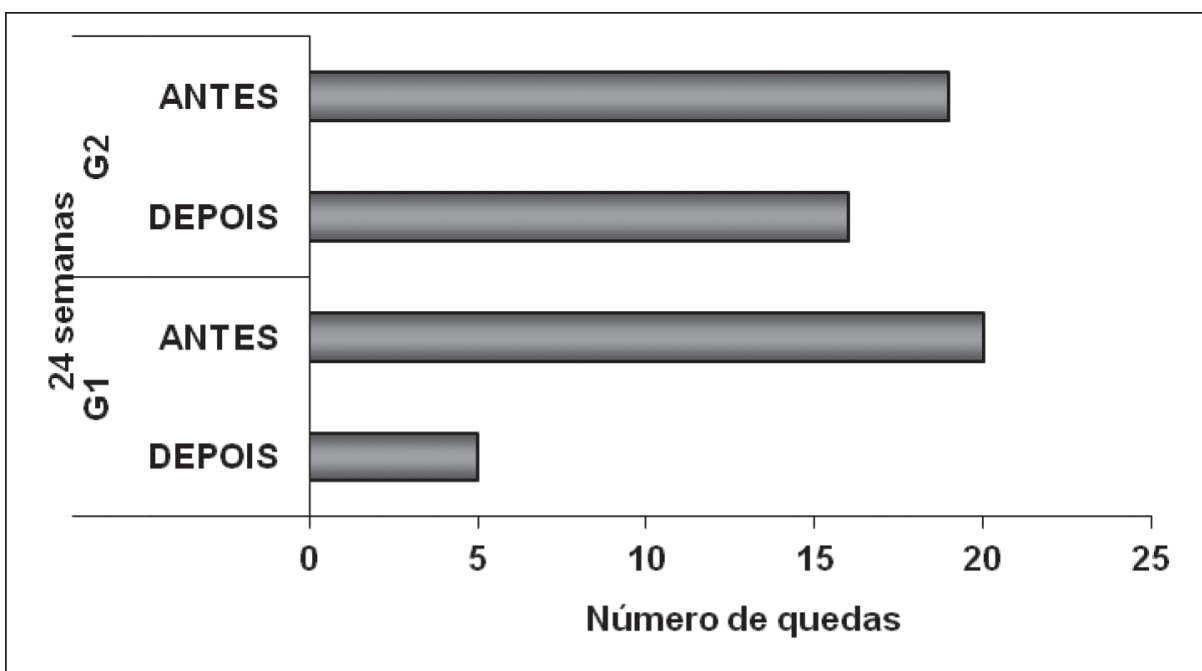

Figura 3 - Número de quedas nas 24 semanas anteriores ao tratamento (antes) e após as 24 semanas de tratamento (depois), nos grupos intervenção (G1) e controle (G2). São Paulo-SP, 2007-2009.

\section{DISCUSSÃO}

A Sociedade Brasileira de Geriatria e Gerontologia, em sua publicação intitulada Quedas em Idosos: prevenção, deixa uma questão importante a ser respondida: como selecionar a subpopulação de idosos mais elegível para cada tipo de intervenção? ${ }^{18}$ Desta forma, o modelo proposto por Shumway-Cook et al., ${ }^{16}$ aplicado por este grupo e também por outros autores, pode ser uma ferramenta importante para identificar a população mais vulnerável a quedas e suas consequências.
Poucos estudos levam em consideração a importância do treinamento proprioceptivo como parte fundamental e indissociável de um programa de fortalecimento muscular. Mecanorreceptores localizados nas articulações, tendões, músculos e tecidos adjacentes dão ao sistema nervoso informações sobre posição e movimentos articulares e sobre as forças produzidas nos músculos. ${ }^{20,21}$

A propriocepção do joelho é essencial para modulação e ativação precisa da contração muscular, uma vez que a habilidade funcional 
e o equilíbrio muscular são fortemente afetados pela inexatidão proprioceptiva e pela fraqueza muscular. ${ }^{22}$ Estudos em pacientes com lesões ligamentares do joelho mostram que o treinamento proprioceptivo promove uma informação sensorial adicional, que contribui para a melhora do desempenho do controle postural. ${ }^{21}$ Essa relação se torna ainda mais importante quando o programa de fortalecimento muscular visa melhorar o equilíbrio funcional e prevenir quedas.

Em pesquisa realizada por Geusens et al., ${ }^{23}$ observou-se maior correlação entre quedas e fraturas do que entre osteoporose e fraturas, sendo que a associação de ambas torna esse risco ainda maior.

Nessa pesquisa, a melhora apresentada no equilíbrio, avaliada pela BBS, apesar de mudanças numéricas pequenas, foi consistente, concordando com resultados encontrados por Madureira et al. ${ }^{24}$

Em estudo controlado e randomizado, Malmors et al. ${ }^{25}$ aplicaram treinamento de 10 semanas de fortalecimento, equilíbrio e alongamento em 53 mulheres com osteoporose pós-menopausa, e observaram melhora significativa da força e massa muscular, além do equilíbrio estático.

Madureira et al., ${ }^{24}$ em um programa de 12 meses de treinamento de equilíbrio associado e exercícios orientados para casa, aplicados a 66 mulheres com osteoporose pós-menopausa divididas em dois grupos, um que recebeu treinamento e outro controle, obtiveram resultados significativos no equilíbrio, mobilidade e redução do número de quedas.

Swanenburg, et al. ${ }^{26}$ aplicaram três meses de treinamento de força, equilíbrio e coordenação em 24 mulheres com osteoporose ou osteopenia com 65 anos ou mais. Após 12 meses, observaram redução do risco de cair (Escala de Equilíbrio de Berg), e aumento da força muscular de membros inferiores, culminando com a redução do número de quedas no grupo intervenção (89\%).

Shumway-Cook et al. ${ }^{16}$ avaliaram o modelo de previsão quantitativa de quedas em comparação com o histórico de quedas nos últimos seis meses, examinando a sensibilidade e especificidade. A sensibilidade encontrada foi de $91 \%$ e a especificidade, $82 \%$, onde 20 dos 22 pacientes com histórico de quedas foram classificados corretamente pelo modelo e 18 dos 22 pacientes dos pacientes sem histórico de quedas foram corretamente classificados.

Neste estudo, o modelo utilizado para previsão quantitativa do risco de quedas mostra redução significativa do risco de cair, concordando com a utilização do modelo feita por Resende et al. ${ }^{27}$ Foram encontradas sensibilidade de $81 \%$ e especificidade de $78 \%$, ao ser comparado o número de quedas durante o período de seis meses, o que corrobora o encontrado por Shumway-Cook et al. ${ }^{16}$

Possível limitação que pode ser verificada neste estudo é a aplicação de testes e escalas funcionais, que, apesar de serem validados, não têm a precisão dos testes de laboratório considerados "padrão ouro". Por outro lado, a utilização da Escala de Berg (aplicação do índice de quedas) é altamente reprodutível na prática clínica diária, onde o acesso aos testes laboratoriais é pouco frequente.

\section{CONCLUSÃO}

A associação do treinamento sensório-motor e da força progressiva para quadríceps é eficaz na prevenção de quedas e redução de seus fatores de risco. $\mathrm{O}$ modelo para previsão quantitativa do risco de quedas pode ser útil na identificação do risco de quedas em pessoas idosas. 


\section{REFERÊNCIAS}

1. Riera R, Trevisani VFM, Ribeiro JPN. Osteoporose - A importância da prevenção de quedas. Rev Bras Reumatol 2003;43(6):364-8.

2. Tinetti ME, Williams CS. Falls, injuries due to falls, and the risk for admission to a nursing home. $\mathrm{N}$ Engl J Med 1997;337(18):1279-84.

3. Campbell AJ, Robertson MC, Gardner MM, Norton RN, Tilvard MW, Buchner DM. Randomised controlled trial of a general practice programme of home based exercise to prevent falls in elderly women. BMJ 1997;315(7115):1065-9.

4. Secretaria de Estado da Saúde de São Paulo. Vigilância e prevenção de quedas em pessoas idosas. São Paulo: SES/SP, 2010. [acesso em 16 jan 2013]. Disponível em: < http://www.saude.sp.gov.br/ resources/ccd/publicacoes/publicacoes-ccd/saude-epopulacao/35344001_site.pdf

5. Weatherall M. Prevention of falls and fall-related fractures in community-dwelling older adults: a metaanalysis of estimates of effectiveness based on recent guidelines. Intern Med J 2004;34(3):102-8.

6. Pils K, Neumann F, Meisner W, Schano W, Vavrovsky G, Van der Cammen TJ. Predictors of falls in elderly people during rehabilitation after hip fracturewho is at risk of a second one? Z Gerontol Geriatr 2003;36(1):16-22.

7. Johnell O, Kanis JA, Oden A, Johansson H, De Laet C, Delmas P, et al. Predictive value of BMD for hip and other fractures. J Bone Miner Res 2005;20(7):1185-94.

8. Siris ES, Brenneman SK, Barrett-Connor E, Miller PD, Saijjan S, Berger ML, et al. The effect of age and bone mineral density on the absolute, excess, and relative risk of fracture in postmenopausal women aged 50-99: results from the National Osteoporosis Risk Assessment (NORA). Osteoporos Int 2006;17(4):565-74.

9. Cólon-Emeric C, Kuchibhatla M, Pieper C, Hawkes W, Fredman L, Magaziner J, et al. The contribution of hip fracture to risk of subsequent fractures: data from two longitudinal studies. Osteoporos Int 2003;14(11):879-83.

10. Colón-Emeric CS, Saag KG. Osteoporotic fractures in older adults. Best Pract Res Clin Rheumatol 2006;20(4):695-706.

11. American College of Sports Medicine; ChodzkoZaiko WJ, Proctor DN, Fiatarone Singh MA, Minson CT, Nigg CR, et al. American College of Sports Medicine position stand. Exercise and physical activity for older adults. Med Sci Sports Exerc 2009;41(7):1510-30.
12. Teixeira LE, Silva KN, Imoto AM, Teixeira TJ, Kayo AH, Montenegro-Rodrigues R, et al. Progressive load training for the quadriceps muscle associated with proprioception exercises for the prevention of falls in postmenopausal women with osteoporosis: a randomized controlled trial. Osteoporos Int 2010;21(4):589-96.

13. Robertson MC, Devlin N, Gardner MM, Campbell AJ. Effectiveness and economic evaluation of a nurse delivered home exercise programme to prevent falls. 1: Randomised controlled trial. BMJ 2001;322(7288):697-701.

14. Robertson MC, Gardner MM, Devlin N, McGee R, Campbell AJ. Effectiveness and economic evaluation of a nurse delivered home exercise programme to prevent falls. 2: Controlled trial in multiple centres. BMJ 2001;322(7288):701-4.

15. Carter ND, Kannus P, Khan KM. Exercise in the prevention of falls in older people: a systematic literature review examining the rationale and the evidence. Sports Med 2001;31(6):427-38.

16. Miyamoto ST, Lombardi Junior I, Berg KO, Ramos LR, Natour J. Brazilian version of the Berg balance scale. Braz J Med Biol Res 2004;37(9):1411-21.

17. Berg K, Norman KE. Functional assessment of balance and gait. Clin Geriatr Med 1996;12(4):705-23.

18. Shumway-Cook A, Baldwin M, Polissar NL, Gruber W. Predicting the probability for falls in communitydwelling older adults. Phys Ther 1997;77(8):812-9.

19. American College of Sports Medicine Guidelines for Exercise Testing and Prescription. 5th. Baltimore: Lippincot Williams \& Wilkins; 1995. 366 p.

20. Pereira SRM, Buksman S, Perracini M, Py L, Barreto KML, Leite VMM. Quedas em idosos: prevenção. Projeto Diretrizes. Associação Médica Brasileira e Conselho Federal de Medicina. 2008 [em 16 jan 2013]. Disponível em: http://www.projetodiretrizes.org.br/ projeto_diretrizes/082.pdf

21. Hurley MV. Muscle dysfunction and effective rehabilitation of knee osteoarthritis: what we know and what we need to find out. Arthritis Rheum 2003;49(3):444-52.

22. van der Esch M, Steultjens M, Harlaar J, Knol D, Lems W, Dekker J. Joint proprioception, muscle strength, and functional ability in patients with osteoarthritis of the knee. Arthritis Rheum 2007;57(5):787-93.

23. Bonfim, TR, Grossi DB, Paccola CA, Barela JA. Additional sensory information reduces body sway of individuals with anterior cruciate ligament injury. Neurosci Lett 2008;441(3):257-60. 
24. Geusens P, Autier P, Boonen S, Vanhoof J, Declerck $\mathrm{K}$, Raus J. The relationship among history of falls, osteoporosis, and fractures in postmenopausal women. Arch Phys Med Rehabil 2002;83(7):903-6.

25. Madureira MM, Takayama L, Gallinaro AL, Caparbo VF, Costa RA, Pereira RM. Balance training program is highly effective in improving functional status and reducing the risk of falls in elderly women with osteoporosis: a randomized controlled trial. Osteoporos Int 2007;18(4):419-25.

26. Malmros B, Mortensen L, Jensen MB, Charles P. Positive effects of physiotherapy on chronic pain and performance in osteoporosis. Osteoporos Int 1998;8(3):215-21.

27. Swanenburg J, de Bruin ED, Stauffacher M, Mulder T, Uebelhart D. Effects of exercise and nutrition on postural balance and risk of falling in elderly people with decreased bone mineral density: randomized controlled trial pilot study. Clin Rehabil 2007;21(6):523-34.

28. Resende SM, Rassi CM, Viana FP. Effects of hydrotherapy in balance and prevention of falls among elderly woman. Rev Bras Fisioter 2008;12(1):57-63. 